\title{
A communication architecture for multi-agent applications in FMS/FAS
}

\author{
J. Reinaldo Silva ${ }^{*}$ ) \\ University of São Paulo \\ Rua Prof Mello Morais, 2231 \\ São Paulo - Brazil \\ Tel.: +55-11-818-5545, fax: +55-11-818-5471 \\ e-mail: jorsilva@usp.br
}

\begin{abstract}
A communication architecture is presented as well as a model for a language and protocol based in Speech Acts, which provides a framework for agents in a manufacturing environment. The model can be formalized by using Fifo nets, what can be represented by Petri Nets or a suitable extension called PFS/MFG, which introduces abstract elements. We claim that the resulting system can be useful to the design and modeling of agent society in the manufacturing environment balancing the complexity of the communication between language and protocol.
\end{abstract}

\section{Keywords}

Multi-agents, communication protocol, fifo nets, PFS/MFG

\section{INTRODUCTION}

Multi-agent systems (MAS) have being presented as a new step in the area of Artificial Intelligence, combining logical assets with open distributed systems. Besides being a very interesting theoretical subject to study, MAS have being pointed as a good framework for new developments in Flexible Manufacturing Systems (FM S) and Factory Automation Systems (FAS) (Jennings, 1994) (Camarinha-Matos, 1995).

Flexible systems, which have to change its control program from time to time, that share resources, tools, and eventually use some idle machine in another cell, can profit a lot from the flexibility of a multi-agent approach. Balanced automated systems can be formalized as a society where some of the agents involved are humans (once a reasonable model for their action is provided). However there are some drawbacks. Multi-agent systems are known by the overhead of computation necessary to

(*) Partially supported by CNPq and Flexsys project, ECLA-76101. 
coordinate its action, to communicate, or to solve conflicts. Besides, formal aspects of coordination and communication are still an open discussion.

In this work we propose a very simple approach to agent communication suitable to the manufacturing environment. The approach is based in the principle that the target system should be prepared to face new trends in virtual factory network but should avoid as much as possible to base coordination in the exchange of messages. Communication messages, by its turn, are based on a simple set that can be described by Speech Acts (Werner, 1987) and illocutionary forces (Sing, 1992). The final communication language is application oriented and can be formally described with FIFO nets (a Petri Net extension) that can be mapped in PFS/MFG (another Petri Net extension with abstract elements). This formal assets provide a sound framework to formal specification and design of communication to a manufacturing society of agents.

Section 2 is devoted to the description of the role of Speech Acts and illocutionary forces. Section 3 presents the current state of our work implementing a communication testbed, implemented in an agent society dedicated to intelligent routing, the DCS System (Ghenniwa, 1995). Section 4 points to a formalization of the communication strategy using and extended Petri Net called PFS/MFG.

\section{THE ILLOCUTIONARY PROTOCOL}

Speech Acts were defined in the original work of (Searle, 1969) as an expression which meaning consists of two parts: a referential component and an illocutionary force. The first is connect with the semantics of the message while the second indicate the class of the response.

Generally a message is presented as $\mathrm{M}=\langle F(p), p\rangle$, where $p$ represents the referential content and $F(p)$ its illocutionary force (Werner, 88). We can then divide messages and its utterance in classes. If we are not interested in human speech (or at least not in natural language speech), we can imagine that a small set of illocutionary forces would be enough to cover all utterances connected with a given application.

Thus, it is possible to define a pragmatic operator capable to associate each illocutionary force to a set of actions that a given agent is capable to do, simplifying the interpretation process. Specific illocutionary forces can act in different forms over agents changing its knowledge, intention or strategy.

Using the formalism introduced by Werner (Werner, 89) we identify a discourse situation $d$ where speech acts $\langle s, a, h\rangle$ are performed by an speecher $s$ and a hearer $h$ by sending a message sentence $a$. from $s$ to $h$. Each agent (speecher and hearer) is characterized by a relational state $R=\langle I, S, V\rangle$ where $I$ is the informational state (an structure composed of all the information the agent has), $S$ is the intentional state which, for a given informational state, can give all actions that could be taken in he current state that are consistent with the agent intention. Finally $V$ is the evaluative state which is given by a set of evaluation functions that gives the probability that a course of action be selected.

We define the $\operatorname{Prag}(a)$ operator which for a given illocution takes all the actions the sentence $a$ suggest. 
In this work we use only four illocutionary forces defined as,

1. Assertive : An information is included in the message $a$ so that, if $I_{h}, I_{s}$ and $I_{s}^{h}$ are the information state of the hearer, of the speecher and the hearer model in the speaker beliefs,

$$
\begin{aligned}
& I_{h} \stackrel{a}{\longrightarrow} \operatorname{Prag}(a) I_{h} \\
& I_{s}^{h} \stackrel{a}{\longrightarrow} \operatorname{Prag}(a) I_{s}^{h}
\end{aligned}
$$

meaning that both the information state of the hearer and the model of the hearer in the speecher belief are modified by the appearing of sentence $a$, and by the occurrence of all pragmatic actions generated by that.

2. Directive: A direct command is included in the message $a$ so that, if $S_{h}, S_{s}$ and $S_{s}^{h}$ are respectively the intentional state of the hearer, the intentional sate of the speaker and the speaker's representation of the hearer new intentions,

$$
\begin{aligned}
& S_{h} \stackrel{a}{\longrightarrow} \operatorname{Prag}(a) S_{h} \\
& S_{s}^{h} \stackrel{a}{\longrightarrow} \operatorname{Prag}(a) S_{s}^{h}
\end{aligned}
$$

meaning that a command would alter the strategy of the hearer and the model in the speaker of the hearer's strategy (if it is explicitly represented in the agent architecture).

3. Commissive: If a commission is included in the message $a$ then

$$
\begin{aligned}
& S_{s} \stackrel{a}{\longrightarrow} \operatorname{Prag}(a) S_{s} \\
& S_{h}^{s} \stackrel{a}{\longrightarrow} \operatorname{Pr} a g(a) S_{h}^{s}
\end{aligned}
$$

where the sentence alter the intentional state of the speaker and the model in the hearer of the speaker's new beliefs.

4. Permissive: In this work we distinguish in the commissive illocution the permissive messages, that is, those asking and granting permission to use a shared resource currently in possession of the hearer. In such case the asking sentence alter the hearer's representation of the speaker new intention, and the answer of the hearer can (but not necessarily will) change the strategy of the speaker. The difference from the previous illocution is that the effect is achieved by two messages, $a$ and $\bar{a}$ (the answer to $a$ ) and that the permission will not necessarily alter the intentional state of the speaker.

\section{IMPLEMENTATION IN DCS SYSTEM}

The DCS (Distributed Coordination System) (Ghenniwa, 1995) was conceived to give some feedback in the behavior of multi-agent societies to handle flexible applications. An important characteristic of the system is the role played by rationality to avoid that "agents spend more time thinking what to do then actually doing it" (Smith, 1980). Rationality is a good strategy for an agent to capture its role in the society as well as other agents intentions. Generally, a rational agent would take a shorter time to decide what to do than a classical intelligent agent. Consequently, they would require a smaller number of messages to offer or request service.

DCS Rational System uses an statistical model to relate actions and situations and a very simple communication protocol and language based in first order logic and 
Speech Acts. A reason to select this approach instead of a sophisticated language as KQML is that FMS applications are very heterogeneous, i. e., sometimes require a sophisticated communication and sometimes a very simple one. Our approach would take some work to implement the protocol and the language, but would has the advantage that the final result would have only the complexity required by the application. This is a key feature to have efficient tools based in multi-agents in FMS/FAS.

Another important point is that the complexity of communication is distributed between the complexity of the protocol and the language. The model presented here is a testbed towards the synthesis of a communication protocol and language which complexity is balanced between then.

The application addressed in DCS system is an intelligent information network where data is transferred by request from point to point by routing the best access path. The architecture of the system is showed in Figure 1 bellow.

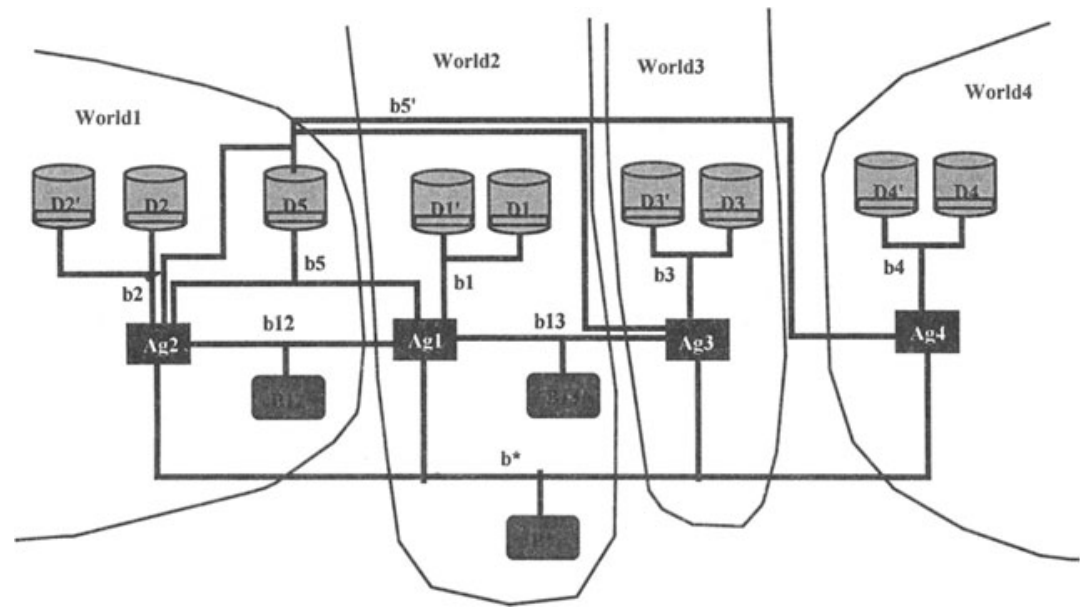

Figure 1

In Figure 1 the data storage $D_{1}, D_{1}^{\prime} \ldots D_{5}$ are accessed by logical channels $b_{1} \ldots b_{5}$. Intermediary buffers $B_{12}, B_{13}$ and $B^{*}$ can be reached by channels $b_{12}, b_{13}$ and $b^{*}$ respectively, which can also be used for the communication between agents. Thus, logical channels are shared resources in the communication process and in the routing process as well.

The system has four partitions each of which containing one intelligent agent and several data storage (active databases) as well as buffers, and local channels. We call each of this partitions a world. A world can be hosted by a computer or distributed cluster of workstations. In the current implementation each world is hosted by a workstation.

Each agent should hold a modular communication package which architecture is showed in Figure 2. 


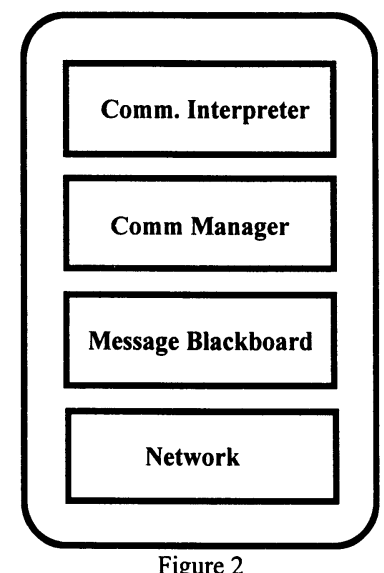

The first level from the bottom is the physical network level. In the original implementation the NIS system was used as a local network just to abstract DCS implementation from low level aspect of communication. A socket system is now being developed to replace it.

The second level is composed of two blackboards (in fact queues) where all messages addressed to a given world or leaving the world are stored, respectively. The communication manager is responsible for opening the message frame and getting the prepositional message.

A message frame is composed of eight different slots,

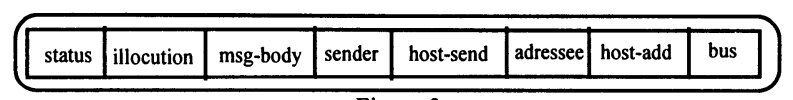

Figure 3

The role of the communication manager is just to deliver the message to the correct addressee inside the world (if it was correctly addressed), and prepare the answer if it one is expected. It is possible to predict if an answer should be expected or not by the illocution. Thus, the communication manager owns a model of the communication and part of the language, consisting of the illocution tokens.

Finally, the communication interpreter has to receive the message contents and determine which actions it must start.

A simple protocol was implemented to the routing system showed in Figure 1, based on four basic illocutionary tokens: directive, assertive, comissive and permissive.

Directive illocution covers the kind of message that relies on the fact that all agents are eagle to cooperate and will do it except if it is committed to some other task or if it is out of its capability.

Assertive messages denotes the intention of doing something or informing another agent about eventual change in one agent beliefs.

Comissive messages denotes the acceptance of a task or sub-task with or without conditions (time conditions or dependencies).

Permissive messages stand for an answer to a request (or intention to use some resource attributed to another agent enclosed in an assertive message). 
In DCS application a very simple language was chosen, consisting of a limited set of tokens and precedence rules based on low level actions: read, write, clear, empty, free, and authorize. The two first actions are self-explanatory. Clear means that one agent commands a buffer or a database to clear part of its storage. Empty is an assertion asking an agent to erase all data in a buffer or database. Free is a commissive term and means that some agent will release a buffer it is currently assigned to use. Finally, authorize is a permissive message asking for authorization to read a storage element.

\section{A PETRI NET REPRESENTATION FOR COMMUNICATION}

There are two more important aspects in multi-agent development: the amount of knowledge about the society one agent has to store, and the possibility of studying specific behaviors of agents and the availability of resources in the early phases of design. Both ideas point to a formal representation of agents relationships, dependencies, and also communication followed by a verification and simulation process.

As we have seen, DCS system is divided in worlds composed of agents, resources (logical channels) and active databases and buffers. Each world has a message package which first duty is to store received messages in a queue and redistribute afterwards. Thus, the communication package of the model shown in Figure 1 can be represented by a net as depicted in Figure 4.

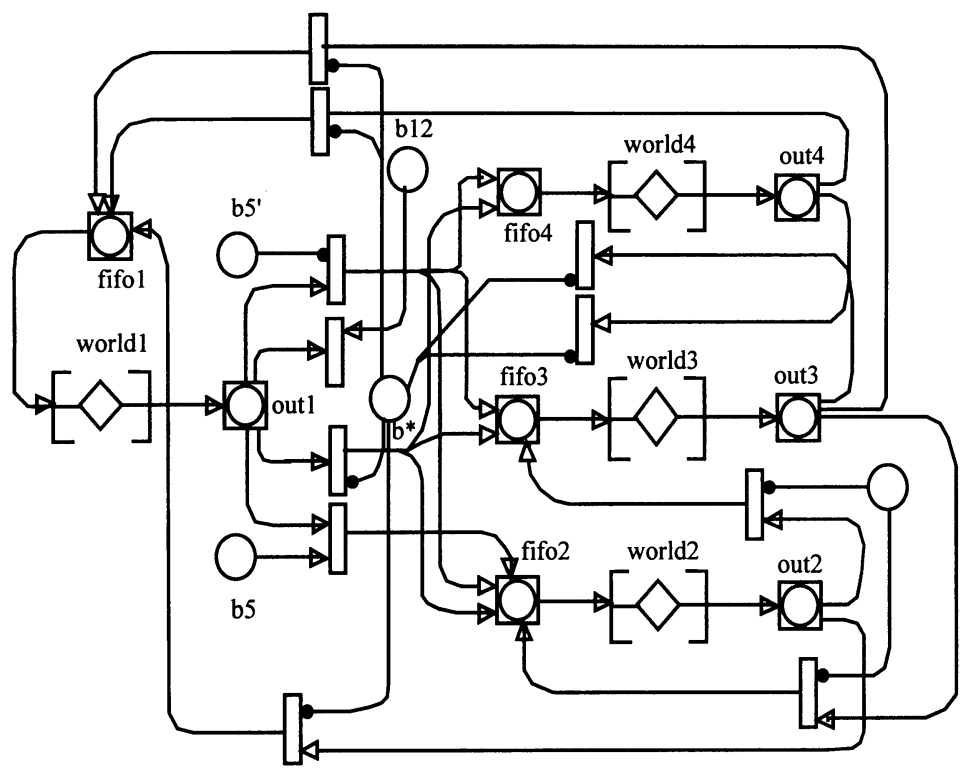

Figure 4 
New achievements in PFS/MFG formalism allow us to use a less cumbersome representation where the resources (logical channels) can be represented by mark attributes similarly to those in colored Petri Nets (Silva and Miyagi, 1996)

Besides expressibility, the advantage of using abstract elements as activities (the composed elements with a losangle in the middle) to represent worlds. The internal behavior and relation of the world are encapsulated and it could be treated as a black box where messages are delivered or received through the message system.

Another important aspect is that by using Fifo Nets implemented by PFS/MFG we introduce a simple way to not only produce rational agents but also rational agent communication, in the sense that agents can use the formalism of nets to save some overhead. Besides, we have the guarantee that no messages will be lost or duplicated, and that, for a specific channel they will arrive in the same order they were emitted. That fact saves some work in handling messages (Fachon, 1992).

\section{CONCLUSION}

The proposal presented here is in fact a design strategy of multi-agents systems in the domain of automated systems. Our proposal is based in two strong points: that MAS are a very good approach to flexible system, mainly if we intend to construct balanced systems where we include in the model the participation of human agents. Another point is the perspective of combining different architectures and communication strategies.

A top down refinement approach was presented based in a case study: of the DCS system. Such approach allow the use of an heuristic search for a good balance between the complexity of the protocol and the complexity included in the communication system. We claim that this approach can lead to a better pay-off than applying complex languages and general frameworks, at least for flexible systems.

\section{REFERENCES}

Camarinha-Matos, L., Afsarmanesh, H. and Silva, J.R. (1995) Multi-Agents in FMS/FAS, IFAC Symposium in Low Cost Automation, Buenos Aires.

Fanchon, J. (1992) A Fifo-Net Model for Process with Asynchronous Communication, Lect. Notes in Computer Science, 609.

Ghenniwa, H., Silva, J.R., Kamel, M. (1995) Distributed Coordination: A Rational Approach, 14th. IJCAI, Workshop in Distributed Information Networks, Montreal.

Hewitt, C. (1991) Open Information System Semantic for Distributed Artificial Intelligence, Artificial Intelligence, 47.

Singh, M.(1991) Toward a Formal Theory of Communication for Multi-Agent Systems, Int. Joint Conf. in Artificial Intelligence.

Searle, J.R. (1969) Speech Acts: An Essay in the Philosophy of Language, Cambridge University Press, London.

Silva, J.R. and Miyagi, P.E. (1995) PFS/MFG: A High Level Net to the Design of Flexible Manufacturing Systems, BASYS'95, Vitoria. 
Silva, J.R. and Miyagi, P.E. (1996) A Formal Approach to PFS/MFG: A Petri Net Representation of Discrete Manufacturing Systems, to appear in Studies in Informatic and Control, IT Press.

Smith, R. (1985) The Contract Net Protocol: High Level Communication and Control in a Distributed Problem Solver, IEEE Transactions on Computers.

Werner, E. (1988) Toward a Theory of Communication and Cooperation for MultiAgent Planning, Theoretical Aspects of Reasoning About Knowledge, Proc. of the 2nd. Conf., Morgan Kauffman Pub..

Werner, E. (1989) A Unified Theory of Communication and Social Structure, in Distributed Artificial Intelligence, Research Notes in Artificial Intelligence, Les Gasser and Michael Hunhs (eds.), Morgan Kauffmann Pub., 1989.

J.R.Silva received a Doctor in Eng. degree by the University of São Paulo, a PPhD by the University of Waterloo, Ca., a Ma. by Mills College, USA. His principal interests are in Engineering Design, particularly in the modeling and design of flexible and balanced systems, using formal methods including Petri Nets, IA, and distributed IA. Presently he is an assistant professor at the University of São Paulo, Brazil. 